\title{
APRENDER Y ENSEÑAR FILOSOFÍA EN EL MUNDO CONTEMPORÁNEO: De la mercantilización del pensamiento al despliegue de su ejercicio*
}

\author{
Óscar Pulido Cortés ** \\ Universidad Pedagógica y Tecnológica de Colombia \\ Grupo de investigación: Filosofía, sociedad y educación
}

Creo que la filosofía siempre que estuvo viva fue algo más que docencia y recuento de ideas. Fue vigilancia crítica, territorio del debate, impulso a la fecundidad del pensamiento. En nuestra sociedad el pensamiento está amenazado tanto por las formas de adaptación que se promueven, como por las formas de desadaptación que se producen. Si la filosofía quiere llegar a ser importante, sino no se conforma con un humilde sitio en la división social del trabajo, como especialización inocua en ideas generales, tiene que saberse combativa y afirmarse combatiente. ${ }^{1}$

Estanistao Zuleta

\section{Resumen}

La pregunta por la filosofía en este mundo contemporáneo agobiado por procesos crecientes de mercantilización y globalización puede pensarse inútil y sin dirección concreta, no obstante, la filosofía se convierte en una forma de resistencia, en una línea de fuga, que permite a los sujetos, que entran en contacto con ella, volver sobre el pensamiento, romper su imagen dogmática y, ubicarlo en un plano de la creatividad, la crítica y la heterogeneidad.

Palabras clave: pedagogía y filosofía, enseñanza de la filosofía.

\section{Abstract}

\section{Learning and Teaching Philosophy in the Contemporary World.}

\footnotetext{
* El presente texto hace parte del proyecto de investigación Filosofía, Sociedad y Educación

** Profesor Escuela de Filosofía y Humanidades. Facultad de Ciencias de la Educación. Licenciado en filosofía. Magíster en Filosofía. Magíster en Educación. Coordinador del grupo de investigación. oscar.pulido@uptc.edu.co

${ }^{1}$ Zuleta Estanislao. "Tribulación y felicidad del pensamiento". En: Zuleta Estanislao. Elogio de la dificultad y otros ensayos . Medellín: Hombre Nuevo editores, 2007, p. 37
} 
The question about philosophy in this contemporary world troubled by processes of increasing commercialization and globalization may seem useless and aimless; nevertheless, philosophy becomes a form of resistance, in a line of flight, that allows subjects who come into contact with it to reevaluate their thinking, break its dogmatic image, and relocate it in a field of creativity, critical thinking and heterogeneity.

Key words: pedagogy and philosophy, philosophy teaching

\section{A MANERA DE INTRODUCCIÓN}

La filosofía y su enseñanza se han convertido en un campo de debate y discusión en el mundo académico contemporáneo. Durante largos períodos de tiempo la filosofía ha ocupado un lugar privilegiado en los estudios universitarios formales y también en épocas más recientes en la educación básica y media. El texto describe las implicaciones y compromisos de enseñar filosofía en las nuevas realidades que presenta el mundo contemporáneo. Plantea las restricciones que la sociedad del nuevo capitalismo coloca la ejercicio del pensar y sugiere algunas fragmentaciones que produce en los sujetos sobre todo los más jóvenes. El trabajo tiene como propósito mostrar que la enseñanza de la filosofía es uno de los soportes fundamentales para generar procesos de formación y ejercicio de pensamiento en los niños y los jóvenes. Intenta, desde una perspectiva contemporánea, darle un sitio al saber filosófico como creación y crítica, posicionar la enseñanza y auscultar a qué tipo de niño y joven se enfrenta la misma. Se recogen los aportes conceptuales de Gilles Deleuze y Michel Foucault para definir de que hablamos cuando hablamos de filosofía y los aportes de Walter Kohan, José Luis Pardo y Alberto Martínez Boom para reflexionar sobre su posibilidad de ser enseñada.

¿Desde qué perspectivas y acciones la filosofía puede ser una disciplina que justifique su presencia en currículos formales de educación básica, media y universitaria? Ningún discurso ni tendencia filosófica logran justificarse a sí mismos, es necesario estudiar la maneras como ellos se han constituido a través de ciertos problemas de interés que han abordado de acuerdo con condiciones propias de construcción y emergencia. La filosofía se convierte para los momentos actuales en una poderosa herramienta de interrogación, ruptura de ciertos modelos y órdenes imperantes que han mercantilizado de tal manera el pensamiento y lo han convertido en un instrumento repetidor, controlador y, sobre todo, eficaz y eficiente.

La filosofía y su enseñanza se han convertido en un campo de debate y discusión en el mundo académico contemporáneo. Durante largos periodos la filosofía ha ocupado un lugar privilegiado en los estudios universitarios formales y también, en épocas más recientes, en la educación básica y media. La filosofía ha tenido diversas formas de ser enseñada de acuerdo con las edades, el género y la ubicación en los currículos, es decir, en algunos casos como estudios introductorios o propedéuticos, y en otros, como fundamento de disciplinas específicas. 
La filosofía y su enseñanza presenta una aporía y es la siguiente: ¿el hecho de saber filosofía autoriza al que la sabe para enseñarla, o el que sabe enseñar puede enseñar cualquier saber, en este caso el filosófico? O planteada de manera diferente: ¿es posible aprender filosofía? Y si lo es, ¿qué es posible aprender de ella?: ¿contenidos?, ¿acciones?, ¿normas?, ¿competencias?

El presente artículo tiene como propósito mostrar que la enseñanza de la filosofía es uno de los soportes fundamentales para generar procesos de formación y ejercicio de pensamiento en los niños y los jóvenes. Describe algunas de las condiciones que generan el proceso de mercantilización del pensamiento especialmente en la relación escuela/mundo del trabajo. Intenta, desde una perspectiva contemporánea, darle un sitio al saber filosófico como creación y crítica, posicionar la enseñanza y auscultar a qué tipo de niño y joven ella se enfrenta.

El texto presenta procede con una especie de "fotogramas", es decir, de cortes, de textos que a través de ciertas imágenes quieren que el que lo escucha o lo lea construya una experiencia que le permita organizar en torno de sí imágenes-movimiento de su sitio como enseñanzate o aprendiz de filosofía, o para decirlo más exactamente en la medida que deviene-enseñanzate o deviene aprendiz de filosofía.

\section{FOTOGRAMA 1}

\section{Sobre la mercantilización del pensamiento y la fragmentación de los sujetos}

Las políticas internacionales en educación direccionadas desde los organismos internacionales fueron desplazando paulatinamente la centralidad en la enseñanza por la centralidad en los aprendizajes entendidos "no como la capacidad general de aprender sino como mera adquisición de ciertas competencias determinadas por resultados efectivos",2. De aquí se desprende que se requieren esquemas cognitivos que permitan desde currículos y formas de interacción pedagógica desarrollar aprendizajes denominados significativos y relevantes que permitan acceder de manera efectiva a los códigos de la modernización y se pueda así regular las actuaciones en la sociedad actual. El énfasis y la valoración de las acciones de aprendizaje están destinadas a que las generaciones de latinoamericanos más jóvenes puedan mediante estrategias de conocimiento satisfacer sus necesidades básicas de aprendizaje, así pues, "la estructura y el contenido de las actividades básicas de aprendizaje deben determinarse de manera que proporcionen a todos los niños, jóvenes y adultos los conocimientos, las habilidades, los valores, las aptitudes que necesitan para sobrevivir, para mejorar sus calidad de vida y poder participar de manera plena y responsable en la vida de sus comunidades y de sus naciones, dar ímpetu y adaptarse a nuevas situaciones y seguir aprendiendo, de acuerdo con sus necesidades e intereses particulares"3.

\footnotetext{
${ }^{2}$ MARTINEZ BOOM, Alberto. "La educación en América Latina: de políticas expansivas a estrategias competitivas". En: Revista Colombiana de Educación. No 44, Bogotá Enero-Junio 2003, p 19

${ }^{3}$ UNESCO. Satisfacción de necesidades básicas de Aprendizaje: Una visión para el decenio 1990. Versión final. 1990 , p. 33.
} 
En el marco discursivo del desarrollo las teorías del aprendizaje se presentan como posibilidad de apropiación, consolidación y establecimiento de estrategias ideológicas de poder que han animado en los últimos años las prácticas pedagógicas de los países latinoamericanos. $\mathrm{Al}$ respecto afirma Tedesco:

...las metodologías didácticas, los diseños curriculares, deberían ocupar un lugar central en la construcción de un nuevo paradigma educativo. Pero su lugar -para que sea teóricamente consistente- debe estar articulado con los aportes de las hipótesis macro sociales y culturales, desde las cuales se generan los interrogantes claves que los diseños pedagógicos deben resolver."4

En este sentido, las reformas educativas y los procesos de diseño curricular que se están adoptando en muchos países del mundo y en particular en América Latina tienen como punto de partida y de llegada las teorías del aprendizaje y en especial las tendencias de orden constructivista desde la epistemología, la psicología y la pedagogía. Este conjunto de posibilidades teóricas para abordar la educación y sus procesos de constitución recibe el nombre de pedagogías psicológicas y son aquellas que toman como eje referencial los fundamentos de estirpe psicológica: la sicología evolutiva, sicología del desarrollo infantil, sicología del aprendizaje, sicología educativa y sicología cognitiva ${ }^{5}$. Son pedagogías psi que parecen ejercer un fuerte dominio y aceptación entre los maestros, y además, han fundamentado gran cantidad de reformas educativas con orientación principal hacia el constructivismo pedagógico o psicológico en todas sus manifestaciones y usos ${ }^{6}$. La pedagogía estuvo históricamente más cerca de la filosofía, y en algunos momentos de la historia, la formación era la misma filosofía, pero se desplazó por la insistencia en modelos psicológico-conductuales y constructivistas, a su vez, con la tecnología educativa que integra el conductismo y la administración, las preguntas por le pensar, la experiencia vital, el cuidado, los postulados éticos queden resueltos en ciertos emplazamientos psicológicoempresariales u organizacionales.

Este abandono de la filosofía en la pedagogía y en las movilidades sociales latinoamericanas no sólo es un problema de la exclusión de la filosofía, es un problema también de la filosofía y de su ejercicio. La filosofía misma se ha convertido sólo en discurso repetitivo y falto de sentido para leer con cierta condiciones de racionalidad las realidades contemporáneas cuando currículos y prácticas de aulas se quedan en recuerdos y nostalgias maravillosas de ideas y autores que si respondieron a su época y que de ninguna manera son trasladados como conceptos vivos y profundamente reinventados, contextualizados a la realidad que viven nuestros pueblos y nuestros niños y jóvenes; de igual maneras las matrices eclesiásticas que en nuestro continente han tendido la filosofía y su enseñanza ha hecho que muchos discursos y autores sean sobrevalorados y otros sencillamente desconocidos y descalificados, incluso, en algunos casos, muchas tendencias

\footnotetext{
${ }^{4}$ TEDESCO, Juan Carlos. (1987) El Desafío educativo. Calidad y democracia. Buenos Aires: Grupo editor latinoamericano. 1990, p 38 ss.

${ }^{5}$ Cfr. VARELA, Julia. "El triunfo de las pedagogías psicológicas”. En: Cuadernos de pedagogía, N 198. Diciembre de 1991.

${ }^{6}$ Cfr. SILVA, Tomaz Tadeu. "Las pedagogías Psi y el gobierno del yo en nuestros regímenes neoliberales". En: SILVA, Tomaz Tadeu. Las pedagogías psicológicas y el gobierno del yo en tiempos neoliberales. Sevilla: Publicaciones, M.C.E.P. 2000
} 
filosóficas y muchos grupos dedicados a su estudios y a la producción de conocimiento se han convertido en verdaderas sectas que producen y actúan con dogmas y no con argumentos.

Esta realidad educativa contemporánea refleja en sus discursos y sus prácticas las nuevas formas de la sociedad orientadas por los cambios impulsados por lo que hoy podemos denominar nuevo capitalismo, es decir, la actualización del mismo, en formas educativas pero sobre todo en los espacios de acción laboral desde los cuales escuelas y universidades plantean sus reformas y ajustes curriculares, incluso es común observar como el llamado sector productivo hoy tiene asiento en las definiciones de los contenidos y prácticas que los niños y jóvenes deben asimilar para sobrevivir en la sociedad de hoy. Estas cercanías y complicidades de los sectores políticos de la educación con la empresa han producido, que para el caso del pensar, el filosofar y la humanidades, efectos tan extraños e indignantes como la reducción de los espacios académicos dedicados a la filosofía y la humanidades en colegios y universidades y la sustitución de los mismos por cátedras como emprenderismo, calidad de vida e incluso autosuperación (para el caso de la ética).

Uno de los aspectos centrales en las prácticas del nuevo capitalismo lo constituye la flexibilidad, categoría central en todas las discusiones contemporáneas: empleos flexibles, instituciones flexibles y para no ir más lejos currículos denominados flexibles, moldeables y acomodables a las diversas transformaciones y cambios mediados claro está por la velocidad. La flexibilidad específicamente el empleo flexible "es aquel cuyo oficio carece de toda delimitación rigurosa: no es un zapatero, ni sastre, ni siquiera obrero de una cadena de montaje de automóviles, sino que tiene que ser capaz de hacer cualquier cosa en un período de formación permanente que se identifica con la longitud completa de su vida laboral y a los largo del cual debe estar dispuesto a reciclarse, reformarse redefinirse y reajustarse cuantas veces sea necesario y en la medida en que lo sea"7.

Estas reflexiones nos permiten volver la mirada hacía la educación y la posibilidad que ésta tiene en un mundo renovado, cambiado y deshecho por la incertidumbre, la inestabilidad y la desazón. Una realidad marcada por otra concepción de formación, y en la cual el sitio del saber y del pensamiento se restringe de tal manera que se limita en el manido eslogan "del pensamiento estratégico", que va quedando como la única posibilidad de ser en estas realidades y es decir, la reducción del pensar a las prácticas de gestión, al desarrollo de competencias en términos de habilidades y a las posibilidades de establecer comunicación que convenza a los "clientes" o usuarios de los servicios. Se reduce el pensamiento a la gestión, la comunicación publicitaria y las habilidades, es decir, una mecanización y acomodación del mismo. El sujeto en estas situaciones se fragmenta, se rompe y se relativiza; puede ser cualquier cosa pero nunca llega a ser nada, es potencia no realizada, es un constante flujo y, en el "proceso permanente de actualización (updating) de la mano de obra se prueba que no desemboca jamás en nada actual. De los individuos que ocupan esto empleos potenciales y efímeros habría que decir, por tanto, que son más bien empleados potenciales, trabajadores únicamente virtuales pero no actuales ni reales, permanentemente

\footnotetext{
${ }^{7}$ PARDO, José Luis. La regla del juego. Sobre la dificultad de aprender filosofía. Galaxia Gutenberg. Círculo de lectores 2004, p 416
} 
en formación, y por ende, en irrevocable minoría de edad, incapaces de abandonar la escuela para incorporarse al mundo de los adultos" $"$.

Esta tendencia de la sociedad contemporánea lleva en sí misma una preocupación y es la incorporación de las nuevas generaciones en estas lógicas extrañas y perversas que llevan a los programas educativos a transformar sus preocupaciones en colchas de retazos para intentar a través de acciones prontas responder "velozmente" a las nuevas estrategias.

\section{FOTOGRAMA 2}

\section{La filosofía como crítica y creación}

La filosofía, en su forma más general, se puede ubicar como aquel discurso que simultáneamente produce conceptos y, a través de andamiajes estructurales, ejerce una función de crítica sobre el mundo y la realidad, es decir, la filosofía es un discurso a la vez creativo y crítico; es un encuentro ${ }^{9}$ de conceptos que le permite a ella misma devenircreativa y devenir-crítica. La característica de la filosofía es el efecto que produce, lo creativo y lo crítico se encuentran, se hallan, se capturan, se roban entre sí, se conectan, se producen y se recrean conjuntamente. La filosofía es creativa-crítica y crítica-creativa por función, acción, recorrido y territorio. Desde la dimensión creativa, dice Deleuze:

...la filosofía, con mayor rigor, consiste en crear conceptos... crear conceptos siempre nuevos... El concepto remite al filósofo como aquel que lo tiene en potencia, o que tiene su poder o su competencia, porque tiene que ser creado... Los conceptos no están esperando hechos y acabados, como cuerpos celestes. No hay firmamento para los conceptos. Hay que inventarlos, fabricarlos o más bien crearlos, y nada serían sin la firma de quienes los crean ${ }^{10}$.

La filosofía es la actividad o el arte de trazar, inventar y crear conceptos. Los conceptos son importantes en función de que nos permiten percibir y analizar acontecimientos. La creación afirma la dimensión de lo nuevo en el pensar. ${ }^{11}$ De esta manera, la función del filósofo consistiría en crear conceptos, inventarlos, dejarlos que circulen y convencer de diversas formas a las personas para que recurran a ellos, pero con una vigilancia clara sobre ellos; la filosofía se puede definir como "conocimiento mediante conceptos puros." 12 Entonces, las concepciones sobre la filosofía cambian radicalmente; no podemos decir simplemente que ella es el "amor por la sabiduría", el arte de la reflexión, pues para amar la sabiduría o para reflexionar sobre algún aspecto de la realidad no se necesita ninguna filosofía, y de ninguna manera se puede considerar como simple comunicación, ya que se ubicaría en el ámbito de la opinión y no de los conceptos. "La filosofía no contempla, no

\footnotetext{
${ }^{8}$ PARDO, José Luis. La regla del juego. Sobre la dificultad de aprender filosofía. Galaxia Gutenberg. Bogotá, Círculo de lectores.2004. p. 417

${ }^{9}$ Cfr. DELEUZE Gilles y PARNET C.. Diálogos. Valencia: Pretextos. Tercera edición. 2004, pp. 5-11.

${ }^{10}$ DELEUZE Gilles y GUATTARI. F.¿Qué es la filosofía? Barcelona: Anagrama. 1993, p. 11.

${ }^{11}$ KOHAN Walter y PINEDA Diego. Pensamiento, acción y sensibilidad. La mirada de filosofía para niños. Beta. 2004. P13

${ }^{12}$ Ibídem, p. 13.
} 
reflexiona, no comunica, aunque tenga que crear conceptos para estas acciones o pasiones"13.

La creación del concepto implica siempre una singularidad, un acontecimiento; todo concepto tiene su historia, aunque transite por diversos planos. En el concepto se encuentran trazas y esquirlas de otros conceptos que en su momento pertenecían a otros problemas y a otros planos. Del mismo modo, todo concepto tiene su devenir, es decir, se sitúa en un mismo plano con otros conceptos, alcanza allí su zona de vecindad, de cercanía, de indiferenciación, y es, a su vez, capaz de diferenciar y darle contenido a una determinada población. ${ }^{14}$ Los conceptos se concatenan, se coordinan, pertenecen a un núcleo común de problemas, pertenecen al mismo borde filosófico, a la frontera, incluso si proceden de construcciones históricas diferentes, el concepto coexiste con otros en la medida que se plantean problemas se cruzan y entrecruzan, y que constituyen otras regiones de un mismo plano.

Los conceptos son complejos, cada uno remite a otros, no solamente en su historia y su devenir, sino también en los diferentes tipos de conexiones que establecen. Cada concepto tiene diversos componentes, que incluso pueden ser tomados como otros conceptos, pero a su vez, el concepto vuelve inseparables los conceptos dentro de sí. Así, cada uno de los conceptos es "considerado punto de coincidencia, de condensación o de acumulación de sus propios componentes"... "todo concepto remite a otros conceptos, no solo en su historia, sino en su devenir y en sus conexiones actuales" ${ }^{\prime \prime}$. Los conceptos se convierten en la caja de herramientas con la cual trabaja la filosofía; los conceptos hacen que la filosofía sirva, funcione y que encuentre personas que se sirvan de ella. Desde los conceptos, la filosofía se convierte en una coexistencia de planos de orden conceptual y no en una sucesión de sistemas; es historia, pero a la vez, una geográfica superposición de planos: un diagrama, ${ }^{16}$ es creativa y crítica.

La dimensión crítica de la filosofía aparece en algunos creadores de conceptos que han puesto el acento de su trabajo en la posibilidad de subvertir los órdenes del pensamiento que se han convertido en hegemónicos y dogmáticos, aspirando a plantear que la verdad no existe por sí misma como origen y esencia. Los filósofos se pueden clasificar en edificadores (creadores) y sísmicos (críticos); en los dos casos los conceptos se convierten en movimiento y vehiculizan la creación y la crítica; la creación deviene crítica y la crítica deviene creación. Desde la perspectiva crítica, la filosofía ha de ubicarse no como la retransmisora de algunos contenidos de pensamiento, es decir, se trata de optar por la

\footnotetext{
${ }^{13}$ Ibídem, p. 12.

${ }^{14}$ DELEUZE. Gilles. Crítica y Clínica. Barcelona: Anagrama. 1996, p. 12.

${ }^{15}$ Ibídem, p. 23.

16 “... un diagrama es un mapa, o más bien una superposición de mapas. Y entre un diagrama y otro se extraen nuevos mapas. Al mismo tiempo no hay diagrama que no implique, al lado de los puntos que conecta, puntos relativamente libres o liberados, puntos de creatividad, de mutación, de resistencia...” DELEUZE, G. Foucault. Barcelona: Paidós. 1998, p. 70.
} 
curiosidad, "no la que busca asimilar lo que conviene conocer, sino la que permite alejarse de uno mismo," 17 en cuanto posibilita pensar distinto, pensar de otro modo.

La filosofía como crítica es una actitud, "una cierta manera de pensar, de decir; una cierta relación con lo que existe, con lo que sabemos, con lo que hacemos, una relación con la sociedad, con la cultura, también una relación con los otros." ${ }^{18}$ El reconocimiento de la actitud crítica hace posible que la filosofía, en las actuales circunstancias, reconozca los límites, las condiciones que bordean el campo de lo que podemos pensar. La crítica como actitud que permite oponerse a estructuras y leyes universales, a la verdad planteada como esencia y exterioridad, a la imagen dogmática del pensamiento; permite oponerse a la adopción de cualquier hábito social como simple costumbre, es decir, como repetición de lo mismo y mecanización de la realidad; y la posibilidad de problematizar las "verdades" y las evidencias que se han construido históricamente y que han sido sublimadas y endiosadas por fuerzas en intereses específicos. La crítica, de igual manera, se opone a cualquier afirmación de una sola y única verdad, para mantener los interrogantes sobre el direccionalidad de lo que hacemos, sobre por qué y para qué hacemos lo que hacemos como prácticas de libertad ${ }^{19}$.

En esta perspectiva, la crítica reúne ciertas características que la filosofía en el mundo contemporáneo debe privilegiar, como la posibilidad de interrogar los modos de gubernamentalidad a los que estamos sometidos, específicamente, a cierto tipo que se puede llamar "neoliberal" y que está produciendo desde la educación nuevos vectores de formación y nuevas formas de subjetividad que carcomen el presente y lo retan. Así, pues, la filosofía en su función crítica se ha de comprender como "el movimiento por el cual el sujeto se atribuye el derecho de interrogar la verdad acerca de sus efectos de poder y al poder acerca de sus discursos de verdad; la crítica será el arte de la inservidumbre voluntaria, de la indocilidad reflexiva. La crítica tendría fundamentalmente como función la desujeción del sujeto, en el juego de lo que se podría denominar, con una palabra, la política de la verdad"20.

La filosofía como crítica, aparte de interrogar, se convierte en creación de nuevas posibilidades de vida, nueva sensibilidad, a través del movimiento del pensamiento, de la resistencia y de las líneas de fuga sobre tantos aspectos y tantos sinsabores que la realidad nos presenta; la acción filosófica como crítica "no consiste en justificar, sino en sentir de otra manera: otra sensibilidad" 21 . Aparece entonces la experiencia como posibilidad de crítica y la crítica como concreción y producción de la experiencia, es decir, la filosofía estudia las condiciones que posibilitan a través de la crítica la novedad, el acontecimiento y la experiencia; produce nuevas y diversas sensibilidades, sentidos y direcciones; formas de existencia activa, que transgreden los órdenes de lo mismo y el sentido común.

\footnotetext{
17. FOUCAULT, Michel Historia de la sexualidad. El uso de los placeres. Edición 14. México: Siglo XXI, 2001 , p.12.

18 FOUCAULT, Michel “QQué es la crítica?”. En: FOUCAULT, Michel. Sobre la ilustración. Madrid: Tecnos. 2003, p. 4.

${ }^{19}$ KOHAN Walter y PINEDA Diego. Op. cit. P 14

${ }^{20}$ FOUCAULT, Michel, ¿Qué es la crítica? Op. cit. p 12

${ }^{21}$ DELEUZE. Gilles. Nietzsche y la filosofía. Anagrama. 1986.
} 


\section{FOTOGRAMA 3}

\section{Sobre enseñar y aprender}

Para comprender el sentido y la dimensión del enseñar, como concepto, se parte de definirlo desde lo que propiamente no es. Enseñar ${ }^{22}$ no es sólo un procedimiento operativo de técnica y forma de transmitir conocimiento; no es la manera como se diseñan y crean nuevos escenarios y ambientes para el aprendizaje, si por ambiente entendemos todo un aparataje técnico lleno de artefactos que interactúan con los estudiantes; no se comprende solamente como la relación afectiva y efectiva de los sujetos involucrados en el acto educativo: maestro-alumno. El enseñar se puede ubicar, más bien, como concepto cercano al pensamiento, como ejercicio artístico. Es decir, como técnica de comprensión y cuidado de sí.

El enseñar, entendido desde esta perspectiva, no es prescriptivo ni procedimental, no se reduce a técnicas de orden didáctico-metodológico, se convierte en un revelar, impulsar, desnudar las certezas del conocimiento construido durante siglos y mostrar sus condiciones de aparición concreta; además, promover la invención o apropiación cada vez más apremiante de conceptos que permitan funcionar en el mundo, pues los conceptos se crean con la única "condición de que satisfagan una necesidad y de que presenten cierta extrañeza, cosa que solo sucede cuando responden a problemas verdaderos. El concepto es lo que impide que el pensamiento sea simplemente una opinión, un parecer, una discusión, una habladuría" ${ }^{23}$. De esta manera, la enseñanza no es una forma estática, por el contrario, circula; es una fuerza que se afirma y que asigna nuevo sentido a los sujetos a quienes interviene. De aquí que:

repensar la enseñanza en dirección hacia el pensamiento es abrir un espacio para la creatividad, la estética y la ética. Es a partir de la consideración del enseñar como posibilitadora del pensar como redefine su dimensión cultural, lingüística, cognoscitiva, artística, y es situando al enseñar en disposición hacia el pensamiento como maestro-alumno, escuela y saber, adquirirán sentido y lugar específico ${ }^{24}$.

Así, el enseñar, desde el maestro o profesor, al niño como Otro válido para aprender, apropiar y usar los conceptos.

Desde esta perspectiva, el enseñar, como forma y expresión del pensamiento, se convierte en una paradójica experiencia que rompe los elementos preestablecidos, formas estatizadas, planeaciones curriculares rígidas y definitivas, formas de control y de restricción del pensamiento, prácticas de saber de dependencia, sumisión y autoritarismo. El enseñar se convierte en un acto del pensamiento azaroso, aventurero, incierto, fugitivo y escurridizo.

Ahora bien, si enseñar es posibilitar el pensamiento y éste es un acontecimiento, es decir, encuentro creativo, el enseñar no pasa ni por la transmisión ni por la repetición, se da en la

\footnotetext{
${ }^{22}$ Cfr. MARTíNEZ, Alberto "La enseñanza como vía del pensamiento". En: ZULUAGA Olga Lucia et al. Pedagogía y Epistemología. Bogotá: Magisterio.

${ }^{23}$ DELEUZE, Gilles. "Sobre la filosofía". En: DELEUZE, Gilles. Conversaciones. Valencia: Pretextos. 1999 , p. 217.

${ }^{24}$ MARTÍNEZ, Alberto. Op. cit., p. 211.
} 
posibilitación y puesta en escena de maneras y formas que permitan el acontecimiento de saber. Si la filosofía asume esta perspectiva de enseñar, surge la pregunta ¿qué es lo factible de enseñar en filosofía?, ¿qué se espera que los estudiantes de filosofía aprendan?, ¿se aprenden contenidos de la filosofía, o la actividad filosófica?

El enseñar remite a la ubicación del maestro como posibilitador del acontecimiento de saber, y el aprender, al estudiante como sujeto de este acontecimiento que es el pensar. Enseñar a pensar se convierte en la principal labor de la filosofía. Pensar crítica y creativamente. Desde esta mirada hay que posponer la transmisión amorfa de contenidos, métodos y caminos conocidos, pues "no hay métodos para enseñar a pensar, no hay respuestas que puedan extrapolar una experiencia de pensamiento. No podemos saber por qué caminos alguien llega a aprender a pensar. Pero sí sabemos que sin lo heterogéneo, sin la diferencia, no hay aprendizaje ni pensamiento" 25 .

En este sentido, si enseñar a pensar no es posible en la relación que alguien le enseña a otro, y si el pensar es encuentro, acontecimiento y posibilidad, el enseñar a pensar en términos creativos y críticos se remite a propiciar el encuentro y el acontecimiento, es decir, propiciar la generación de territorios de saber que permitan tanto al que enseña como al que aprende encontrar y delinear el camino propio como sujeto, su cuidado de sí, sus prácticas de libertad y su formas de relación con el mundo.

\section{FOTOGRAMA 4 \\ Del enseñar filosofía}

Si la filosofía fundamenta su acción en la creación de conceptos, y la enseñanza se plantea como forma del pensamiento, el enseñar filosofía se remitiría no a la creación de los conceptos, sino a la apropiación y enseñanza de estos o, por lo menos, de algunos de ellos que permitan a los niños y jóvenes funcionar en el mundo. Es decir, conceptos que les permitan operar, conocer, comprender las diferentes lógicas que se encuentran instauradas en las sociedades contemporáneas, con realidades cada vez más cambiantes y extrañas.

Realidades donde la velocidad, la imagen, las identificaciones múltiples parecen gobernar la vida. Conceptos que a su vez estructuren maneras diferentes de leer la historia y las tradiciones, no para repetir o simplemente para reproducir modelos y esquemas, sino para lograr todo lo contrario, encontrar el valor del origen y el origen del valor de las construcciones que han realizado los hombres y poder mostrarles que lo que ha sido construido como verdad es susceptible de ser criticado y colocado entre paréntesis y poder re-escribir la historia y a su vez inventar nuevas maneras de configuración, de acción, de resistencia. Conceptos que les permitan estar atentos y tener armas para defenderse en su cotidianidad de los embates cada más fuertes de la sociedad de consumo y, sobre todo, de control, y afirmarse desde la construcción de su subjetividad.

En este sentido el profesor de filosofía, o "el enseñante" de conceptos, necesita ser lo suficientemente versátil, no en términos del mercado y de la flexibilidad que pregonan los

\footnotetext{
${ }^{25}$ KOHAN, Walter. "Educación, filosofía e infancia: caminos para pensar un encuentro". En: Memorias del Congreso latinoamericano para el desarrollo del pensamiento. Bogotá: Fundación Merani, 2002.
} 
paradigmas de la calidad eficientista, sino en términos de la profunda creación y acción a través de prácticas inventadas y reinventadas con los niños y los jóvenes. Esta versatilidad le permite ser archivista, en el sentido de procurar el descubrimiento y la recolección de un número de posibles conceptos con los que pueda trabajar, y cartógrafo, en el sentido de dibujar mapas, encontrar conexiones, acompañar a sus estudiantes a descubrirlos y construirlos, pintar con ellos cuadros.

\section{FOTOGRAMA 5}

\section{¿A quién se enseña la filosofía hoy?}

La nueva arista de este mapa la constituyen las preguntas ¿a quién se le enseña la filosofía hoy? y ¿qué tipo de conceptos se han de privilegiar? Si la filosofía se dedica a pensar y crear conceptos, la pedagogía, además de utilizar la aventura del pensamiento para enseñarla, tiene que hacer la pregunta por el niño y el joven que la aprenden. Esta pregunta remite a conocer, experimentar y describir al niño y al joven de hoy en sus profundas y novedosas dinámicas de vida. Es necesario conocer sus modos de comunicación, sus posibilidades de interrelación.

Las prácticas de los niños y jóvenes de hoy son prácticas de experiencia de orden individual y grupal; donde se reconoce y reconstruye el cuerpo y el espíritu al tiempo; donde el dualismo, otrora determinante en las maneras de ser y de actuar, se desdibuja; donde la paradoja de lo plural es una alternativa a lo individual; donde opuesto a las formas modernas de identidad, los niños y jóvenes de hoy apuestan por "identificaciones múltiples"26, siempre se es otro; esto "implica acudir al juego de las máscaras, la duplicidad donde se encuentra el germen de las resistencias, de las subversiones, de aquellas que no son resistencias políticas, sino que caracterizan las prácticas juveniles" 27 . Este es precisamente el lugar para la diferencia y lo heterogéneo que permite el aprender a pensar.

De otra parte, es importante comprender que el niño y el joven que participan de la clase de filosofía viven situaciones y momentos diferentes en la historia, tienen otros valores, otras prácticas y diferentes formas de relación, de juego, de mirada. El niño y el joven participan de lo que podríamos llamar una cultura neoágrafa, signada por la sucesión y repetición de imágenes y por el cambio en la concepción y comprensión de tiempos y espacios, atravesados por la velocidad. El niño llega a la escuela cargado de saberes, de de muchas inexperiencias. Aprender conceptos y su uso le permitirá al niño transitar caminos con fuertes soportes para afrontar los avatares y vaivenes contemporáneos.

De igual manera es necesario preguntarse cuáles son los objetivos de la educación básica y media, en este caso en Colombia, y qué se pretende enseñar en filosofía, qué tipo de formación filosófica requiere un sistema educativo como el nuestro, que aparentemente centra su atención en valores, actitudes, pensamiento crítico, competencias, que estarían en el campo de la filosofía, que sería el territorio propicio para el acontecimiento del pensar. Enseñar filosofía en la escuela básica y media, con las oportunidades que se están

\footnotetext{
${ }^{26}$ MAFFESOLI, Michel. "Yo es otro". En: Debates sobre el sujeto. Universidad Central. Bogotá, Siglo del Hombre editores, 2003. P 27

${ }^{27}$ Ibídem, p. 27.
} 
presentando, con programas como filosofía para niños y filosofía en el aula, permite a la filosofía ubicarse crítica y creativamente como forma de resistencia y línea de fuga a la mecanización del pensar. El pensar no sólo es un conjunto de habilidades cognitivas, de estrategias para resolver problemas, es también una aventura, una experiencia vital. Enseñar y aprender filosofía se puede convertir, en la escuela básica y media, en una profunda arma de formación política, de respeto y reconocimiento del otro en situaciones tan adversas como las que viven nuestros niños y jóvenes en el mundo contemporáneo.

\section{Bibliografía}

DELEUZE, Gilles. ¿Qué es la filosofía? Barcelona: Anagrama. 1993. . Nietzsche y la filosofía. Barcelona: Anagrama. 1986. . Crítica y clínica. Barcelona: Anagrama. 1996. . "Sobre la filosofía”. En Deleuze, Gilles. Conversaciones. Valencia: Pretextos. 1999. Foucault. Barcelona: Paidós. 1998

Deleuze, G. y Parnet, C. Diálogos. Valencia: Pretextos. Tercera edición. 2004.

Deleuze, G. y Guattari, F. ¿Qué es la filosofía? Barcelona: Anagrama. 1993.

FoucAult, Michel. Historia de la sexualidad. El uso de los placeres. Edición 14. México: Siglo XXI, 2001 . ¿QQué es la crítica?” En: Foucault, Michel. Sobre la ilustración. Madrid: Tecnos. 2003.

KOHAN, Walter. Infancia entre educación y filosofía. Laertes. 2004 . "Educación, filosofía e infancia: caminos para pensar un encuentro". En: Memorias del Congreso Latinoamericano para el Desarrollo del Pensamiento. Bogotá, Fundación Merani. 2002.

Kohan, Walter y PINEDA, Diego. Pensamiento, acción y sensibilidad. La mirada de filosofía para niños. Beta. 2004.

MAFFESOLI, Michel. "Yo es otro". En: Debates sobre el sujeto. Bogotá: 2003.

MARTínEZ, Alberto. "La enseñanza como vía del pensamiento". En: Zuluaga Olga Lucia et al. Pedagogía y Epistemología. Bogotá: Magisterio. 2003.

Obiols, G. y Rabossi, Eduardo. La enseñanza de la filosofía a debate. Buenos Aires: Novedades educativas. 2000.

PARDO, José Luis. La regla del juego. Sobre la dificultad de aprender filosofía. Barcelona: Galaxia Gutenberg, 2004.

Schujman, Gustavo. Filosofía: temas fundamentales y aportes para su enseñanza. Buenos Aires: Biblos. 2007.

ZourABICHVILI, François. Deleuze. Una filosofía del acontecimiento. Amorrortu. 2004. 